\title{
Digital test assembly of truck parts with the IMMA-tool - an illustrative case
}

\author{
L. Hanson ${ }^{\mathrm{a}, \mathrm{b}^{*}}$, D. Högberg ${ }^{\mathrm{c}}$ and M. Söderholm ${ }^{\mathrm{a}}$ \\ ${ }^{a}$ Industrial Development, Scania CV AB, Södertälje, Sweden \\ ${ }^{\mathrm{b}}$ Wingquist Laboratory, Chalmers University of Technology, Göteborg, Sweden \\ ${ }^{\mathrm{c}}$ Virtual Systems Research Centre, University of Skövde, Skövde, Sweden
}

\begin{abstract}
Several digital human modelling (DHM) tools have been developed for simulation and visualisation of human postures and motions. In 2010 the DHM tool IMMA (Intelligently Moving Manikins) was introduced as a DHM tool that uses advanced path planning techniques to generate collision free and biomechanically acceptable motions for digital human models (as well as parts) in complex assembly situations. The aim of the paper is to illustrate how the IPS/IMMA tool is used at Scania $\mathrm{CV} \mathrm{AB}$ in a digital test assembly process, and to compare the tool with other DHM tools on the market. The illustrated case of using the IMMA tool, here combined with the path planner tool IPS, indicates that the tool is promising. The major strengths of the tool are its user friendly interface, the motion generation algorithms, the batch simulation of manikins and the ergonomics assessment methods that consider time.
\end{abstract}

Keywords: digital human modelling, anthropometrics, ergonomics assessment, vehicle manufacturing

\section{Introduction}

Truck design engineers use CAD tools at the design stage to check that parts are without any collision when fixed in their final position. To ensure that the non-collision requirement is satisfied in the final position only is however not enough. There is also a need to check that it is possible to assemble and disassemble the parts. For that reason manufacturing engineers use path planning tools to automatically find collision free motion patterns of parts being moved in space. Such movements typically have a lot of potential obstacles and narrow thoroughfares. These path planning tools detect a path for the part, from a point outside the assembly structure to its final position in the structure, typically without considering any external support required to produce the transfer. With this process the truck industry verifies the design of parts and suggests a suitable assembly sequence. There are commercial path planning tools available on the market today, e.g. from retailers such as Fraunhofer-Chalmers Centre and KineoCam. Within truck cab manufacturing these tools are used for, among other things, investigating possible assembly paths for bed, carpet and seat. The parts can for instance be assembled through the door, sun roof or front window.

In an actual assembly and disassembly context, no part can find its way from one point to another without external support. The part needs to be manoeuvred into its final position by a robot or by a human (or possibly in cooperation). The majority of the parts are assembled or disassembled by humans in a vehicle assembly context.

Several digital human modelling (DHM) tools have been developed for simulation and visualisation of human postures and motions. These tools have typically been used by human factors engineers to analyse human postures adopted when performing assembly tasks. Some DHM tools include human motion generators, based on techniques such as neural networks, root motion modifiers and inverse kinematics. These features are rarely used in industry applications though. However, existing motion generators are able to predict collision free manikin motions, albeit typically applicable in cases where the

\footnotetext{
*Corresponding author. E-mail: lars.hanson@scania.com
} 
environment surrounding the manikin is simple, e.g. where the manikin is able to walk around a table or similar. The automotive assembly environment is however more complex than that.

In 2010 the DHM tool IMMA (Intelligently Moving Manikins) was introduced [1]. IMMA was introduced as a DHM tool that uses advanced path planning techniques to generate collision free and biomechanically acceptable motions for digital human models (as well as parts) in complex assembly situations. Furthermore, the IMMA tool is aimed to be a non-expert tool with high usability, where the tool supports the tool user to consider human diversity, to easily instruct the manikin to perform tasks and functionality to perform time-dependent ergonomics evaluations to control and assess complete motions. The aim of the paper is to illustrate how the IPS/IMMA tool is used at Scania CV AB in a digital test assembly process, and to compare the tool with other DHM tools on the market.

\section{Method}

Scania is a member of the IMMA research and development project carried out in collaboration between academia and other vehicle manufacturers in Sweden. Along the project, demonstration versions of the DHM tool are launched. This is done in order to get feedback and new ideas of future functionality requirements and usability related aspects. The version of the IMMA tool used in this illustrative case is set up to work in combination with the path planner tool IPS (Industrial Path Solutions) developed at Fraunhofer-Chalmers Centre [2].

\section{Result}

Scania uses an established work process that complies with Hanson et al. [3]. In the first step the part or workplace designer describes the assignment by writing the background and purpose of the study to be performed. In this illustrative case a workplace designer has identified that a carpet causes ergonomic problems among the assembly personnel. The simulation engineer, with knowledge of the capabilities of the simulation tool, has agreed to take on the assignment. Together, the workplace designer and the simulation engineer specify the carpet variant of interest, the required resources and the surrounding environment. They also agree on type of result to be delivered and delivery date.

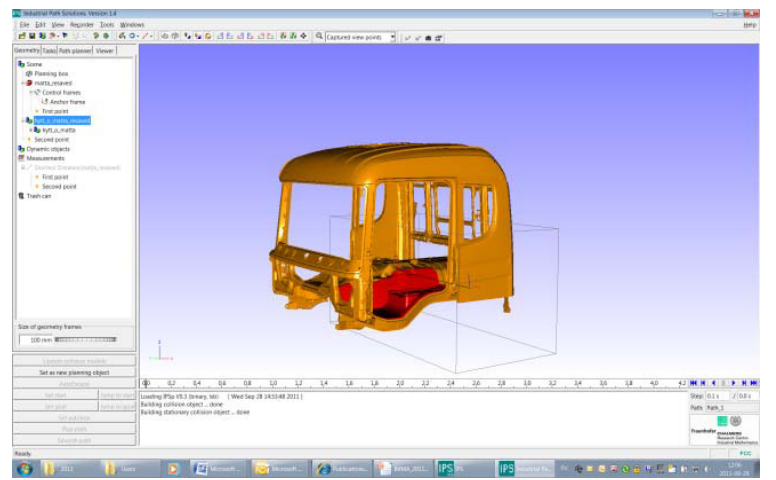

Fig 1. The cab and the carpet loaded in the IPS tool. IPS is used for finding a collision free assembly path for the carpet.

In the second step, the carpet and the surrounding environment are transferred from the PDM system to the IPS tool (Figure 1). IPS is used for finding a collision free path for the carpet. In IPS the carpet is set as the planning object. A collision free start position (inside the cab) is found with functionality for "auto escape" and manual manipulation. With manual manipulation the carpet is moved to an end position outside the cabin. The carpet position is set as end position and an "auto box" is generated to minimise calculation space. With these settings the tool is searching for a collisions free path for the carpet. The result, if existing, is presented as a motion trajectory, sweep volume and shortest distance to surrounding (Figure $2)$. In this simulation a solution was found with a minimum distance to the surroundings of about $6 \mathrm{~mm}$. This means that a collision free path for the object is guaranteed. According to Scania's work process, the next step is to investigate if assembly tools also can find its way through. However, when assembling the carpet no tools are used. Therefore ergonomics was investigated next. 


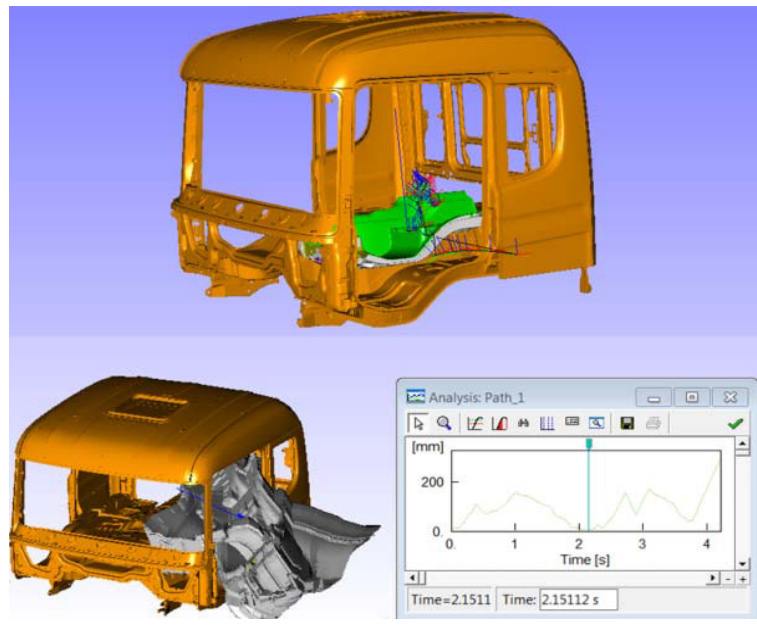

Fig 2. Motion trajectory, sweep volume and shortest distance to the surrounding environment are results from the IPS tool.

As the third step of the work process, the geometry (carpet and cab) is loaded into the IMMA tool. The collision free carpet motion trajectory is loaded as well. Based on information in the assignment, a manikin family is created (Figure 3 ). In this case a Swedish male family covering $75 \%$ of the anthropometric variation in the population was generated. Key anthropometric variables are set as stature and weight. The family generated consists of one manikin representing an average person and four manikins representing extremes on the boundary of the area capturing the chosen accommodation level of the bivariate distribution [4].

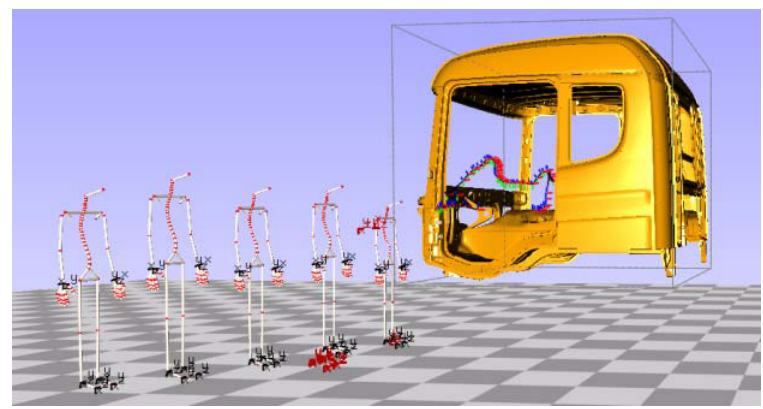

Fig 3. The cab and carpet trajectory together with the manikin family who will test assemble the carpet.

In the fourth step three settings are made: the carpet as planning object, the carpet path trajectory as motion and the manikin family as performer. The weight of the carpet is set to $5 \mathrm{~kg}$. Target points for right and left hand are manually set to grip specific points on the carpet, and the hands are attached to the carpet (Figure 4). The motion generator is then activated [5]. The manikin motion generator creates a human motion where the hands follow the carpet, the biomechanical load acting on the manikins body is minimised and the time in comfortable joint angle zones are maximised, at the same as the manikin is keeping its balance.

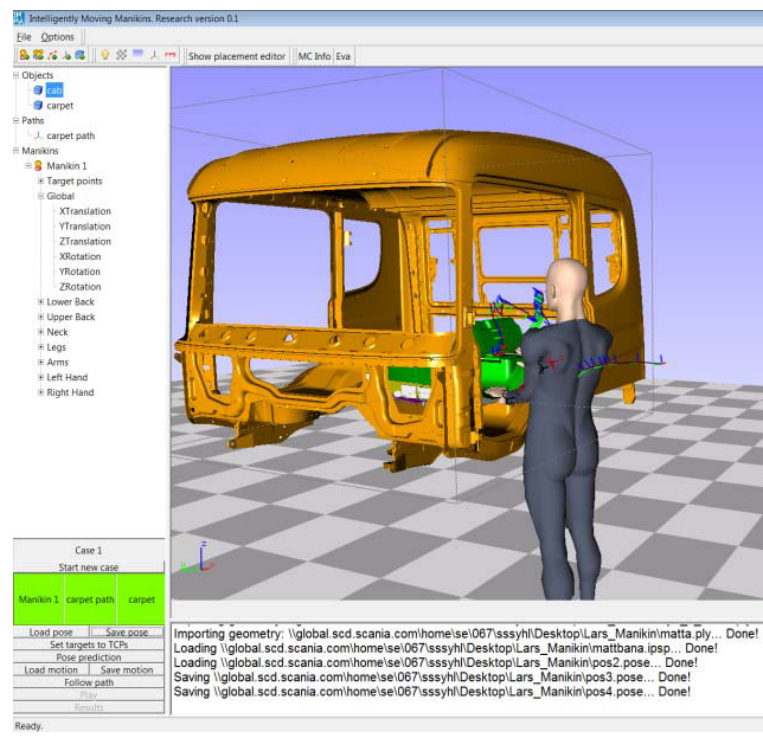

Fig 4. The IMMA tool and a manikin with hands attached to the carpet.

Hand positions and joint angles in the back, neck, shoulder and wrists are recorded during the manikin's motions. This information is then input to the company specific Scania Ergonomic Assessment method. The method indicates that the work posture is acceptable, both for the back, neck, shoulder and wrists (marked green in Figure 5). The method also gives a warning for this assembly task. This is because the manikin is spending too much time outside the recommended working box. The working box is defined as a volume between shoulder and knuckle height as well as elbow span and shoulder grip length. In total the work was rated as acceptable. The IMMA ergonomics assessment toolbox also includes methods that consider time aspects [6]. By using the time based method, more detailed analysis results can be obtained, and in this case it can for example be seen that the wrist is flexed $66 \%$ of the total time. 


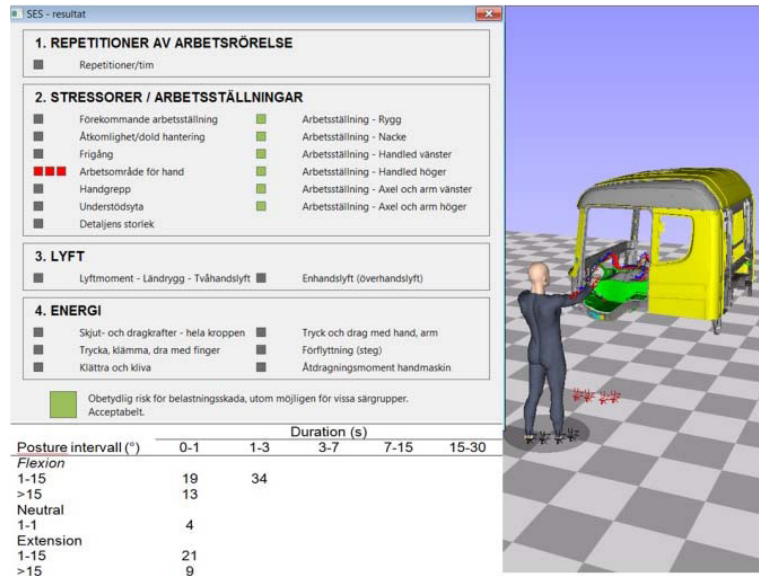

Fig 5. Ergonomics evaluation results for the carpet assembly task. The task is analyzed with Scania Ergonomic Assessment method and with a more detailed time based method for the wrist.

In the sixth and last step the simulation engineer reports the findings to the part and/or workplace designer. In this case the result of the simulation indicates that the carpet is possible to assemble without any collision and that the assembly personnel can perform the task within acceptable ergonomics requirements. To conclude the assignment, a visualization of the manikin performing the carpet assembly task is generated and stored together with the decisions made at the result presentation meeting.

\section{Discussion}

The IMMA manikin is comparable to manikin models in products by Siemens PLM Software and Dassault Systemes. Jack [7], Human Builder and IMMA have similar appearance modes and biomechanical models. The motion generation method in the combined IMMA and IPS tool is comparable to corresponding functionality in the combination of Human Builder and Kineo Path Planner. The case study indicates that the IPS is more user friendly compared to the Catia integrated version of Kineo. The work process is similar for both tools. In both tools the hardest and most time consuming step is to find a collision free starting point. Another time consuming step in Kineo/Human Builder is the positioning of the manikin, with its hands attached to the part, in a natural start position for the assembly.

The IPS/IMMA and Kineo/Human Builder tool combinations are at current status able to generate a collision free way for the part and the tool, which not necessarily is collision free for the human in later steps. The Kineo group has a human path planner in development that will create a collision free path for the part and the human holding the part. The IPS and IMMA tool will be merged in the future. That combined tool will find a smooth collision free motion for the manikin and the part when transferring between different targets in space. The motion generator will consider a set of biomechanical rules to create an ergonomically acceptable motion for the manikin. This means that the mathematical algorithms will find one possible motion for the manikin that is acceptable from an ergonomics perspective. This is a shift in approach. Previously, several laboratories and projects have aimed at simulating natural human movements, e.g. HUMOSIM [8] and ANNIE [9].

The IMMA anthropometric module currently includes one nationality and one age group. The current set-up is able to represent a Swedish working force aged between 18 and 65. Other ergonomics simulation and visualisation tools on the market typically offer more nationalities, more age groups and the consideration of secular trends. To ensure usage at other markets than Sweden, the IMMA tool has a design that makes it easy to add other anthropometric databases. Similarly to Jack and Ramsis [10] the IMMA tool includes methods for the consideration of human diversity when more than one anthropometric measurement is of interest. The anthropometric manikin family generator will be further improved to even better support the tool user to consider anthropometric variation, as well as on how this cause variation in ergonomics evaluation results. For this, the IMMA tool offers a batch simulation feature for running simulations of several manikins performing the simulated assembly motion. This is a similar approach as Ramsis uses for static posture analyses.

There is a lack of confidence in the assessment methods among the users, which usually results in a need for a visualisation of the movement of the manikin. To accomplish that, the user often manually manipulates the manikin to make the visualisation of the movement to look natural. Established DHM tools include ergonomics assessment methods such as RULA [11]. These methods are designed for visual observation of posture analyses. IMMA is in contrast inspired by methods used for analysing and presenting results from technical measurement approaches, e.g. using electromyography (EMG) and electrogoniometers. Based on that philosophy, one detailed analysis method for wrist analysis is modified and integrated in the IMMA tool. Scania's Ergonomic Assessment Method is also integrated in IMMA. It is rather common that companies custom- 
ise the DHM tools they utilise within their organisations, e.g. Volvo Cars has modified eM-Human and Saab Automobile modified IGRIP [12]. This is an indication that companies prefer to rely on their established company specific ergonomics evaluation methods. By integrating usable ergonomics evaluation methods, DHM tools can shift from mainly functioning as visualisation tools, towards acting as advanced simulation tools where objective data is prioritised. The IMMA ergonomics assessment module will be further developed, focusing on assessment methods applied in industry and on methods that consider time factors and the aggregation of ergonomic loads.

The result from the simulation in the illustrative case indicated that a collision free assembly path for the cab carpet existed. However, in the simulation the carpet is rigid. The actual carpet is made of plastic, meaning that in real life it is flexible and foldable. Therefore, it should not be any problem to assemble to carpet from the door opening. In future simulation and visualisation tools it is a challenge to also be able to predict the behaviour for compliant parts.

The result from the assignment indicated no ergonomics risks when performing the work. This task lasts for a couple of seconds and is one part of the work performed at the workplace during a tact (one work sequence). The Scania Ergonomic Assessment method is designed for analysing work tasks with a tact time of more than one minute.

Overall the IPS/IMMA tool is considered as promising. The cooperation between academia and industry employed in the IMMA project is believed to ensure that appropriate science and technology is utilised, and that meaningful results, directly applicable in industry, will be developed. This kind of collaboration is not unique though. Industry, primarily the automotive industry, has sponsored and participated in the development of ergonomics simulation and visualisation tools such as Jack, Ramsis and Human Builder. Still, Sweden is believed to have an exceptionally tight relation between academia and industry that provides for a stimulating environment for developing IMMA as a relevant user friendly industry tool.

\section{Acknowledgement}

This work has been made possible with the support from the Swedish Foundation for Strategic Research/ProViking and by the participating organisations. This support is gratefully acknowledged.

\section{References}

[1] Hanson, L., Högberg, D., Bohlin, R. and Carlson, J.S. (2010). IMMA - Intelligently Moving Manikin - Project Status. Proceedings of 3rd Applied Human Factors and Ergonomics (AHFE) International Conference, Karwowski, W. and Salvendy, G. (Eds.), USA, ISBN 978-0-9796435-4-5.

[2] Carlson J. S., Söderberg, R., Bohlin, R., Lindkvist, L. and Hermansson, T. (2005). Non-nominal path planning of assembly processes. Proceedings of IMECE'05, ASME International Mechanical Engineering Congress \& Exposition, USA.

[3] Hanson, L., Blomé, M., Dukic, T. and Högberg, D. (2006). Guide and documentation system to support digital human modelling applications. Int. J. of Industrial Ergonomics, 36, pp. 17-24.

[4] Bertilsson, E., Hanson, L., Högberg, D. and Rhen, I.M. (2011). Creation of the IMMA manikin with consideration of anthropometric diversity. Proceedings of the 21 st International Conference on Production Research (ICPR), Germany, ISBN: 978-3-8396-0293-5.

[5] Bohlin, R., Delfs, N., Hanson L., Högberg, D. and Carlson, J.S. (2011). Unified solution of manikin physics and positioning. Exterior root by introduction of extra parameters. Proceedings of DHM 2011, First International Symposium on Digital Human Modeling, France, ISBN 978-2-9539515-0-9.

[6] Rhen, I.M., Hanson, L. and Högberg, D. (2011). Risk exposure assessment of dynamic wrist motions of a digital human model. Proceedings of the 43rd annual Nordic Ergonomics Society Conference, Finland, ISBN 978-951-42-9541-6.

[7] Badler, N., Phillips, C.B. and Webber, B.L. (1993). Simulating humans: computer graphics animation and control. New York, Oxford University Press.

[8] Chaffin, D. (2002). Simulation of human reach motions for ergonomics analyses. Proceedings of SAE Digital Human Modeling for Design and Engineering Conference, Germany.

[9] Hanson, L., Akselsson, R., Andreoni, G., Rigotti, C., Palm, R., Wienholt, W., Costa, M., Lundin, A., Rizzuto, F., Gaia, E. Engström, T., Sperling, L., Sundin, A. and Wolfer, B. (1999). ANNIE, a Tool for Integrating Ergonomics in the Design of Car Interiors. SAE Transactions. J. of Material and Manufacturing, Section 5, Vol.108, pp. 1114-1124.

[10]Bubb, H., Engstler, F., Fritzsche, F., Mergl, C., Sabbah, O., Schaefer, P. and Zacher, I. (2006). The development of RAMSIS in past and future as an example for the cooperation between industry and university. Int. J. of Human Factors Modelling and Simulation, Vol.1, No.1, pp. 140-157.

[11] McAtamney, L. and Corlett, E.N. (1993). RULA: a survey method for the investigation of work-related upper limb disorders. Applied Ergonomics, Vol.24, No.2, pp. 91-99.

[12]Högberg, D., Bäckstrand, G., Lämkull, D., Hanson, L. and Örtengren, R. (2008). Industrial customisation of digital human modelling tools. Int. J. of Services Operations and Informatics, Vol.3, No.1, pp. 53-70. 